\title{
Recent Science Highlights from the Keck Interferometer
}

\author{
R. Akeson ${ }^{a}$, M. Colavita ${ }^{b}$, R. Millan-Gabet ${ }^{a}$, S. Ragland ${ }^{c}$, P. Wizinowich ${ }^{c}$, J. Woillez $^{c}$ and the \\ KI team \\ ${ }^{a}$ NASA Exoplanet Science Institute, California Institute of Technology, 100-22, Pasadena, CA, \\ USA; \\ ${ }^{b}$ Jet Propulsion Laboratory, California Institute of Technology, 4800 Oak Grove Dr., Pasadena, \\ CA, USA; \\ ${ }^{c}$ W.M.Keck Observatory, 65-1120 Mamalahoa Hwy, Kamuela, HI, USA
}

\begin{abstract}
The addition of new observational capabilities and continued sensitivity improvements have allowed observations with the Keck Interferometer to encompass new areas of astrophysics and expanded significantly the available sample size in areas which had been the focus of previous work. The technical details of the instrument techniques (including nulling, L-band and increased spectral resolution) are covered in other contributions to this conference. Here, we will highlight the astrophsyics enabled by these instruments including: a summary of the NASA Exo-zodical Dust Survey Key Project, observations across a range of dust temperatures with K and L-band measurements and faint target studies of active galactic nuclei and young stellar disks.
\end{abstract}

Keywords: Infrared interferometry, young stellar objects, active galactic nuclei

\section{KECK INTERFEROMETER}

The Keck Interferometer (KI) combines the two 10-m Keck telescopes as a long-baseline infrared interferometer (see Ragland et al, this volume and references within). It is funded by NASA as a joint development among the Jet Propulsion Laboratory, the W. M. Keck Observatory, and the NASA Exoplanet Science Institute (formerly the Michelson Science Center). KI supports several different observing modes, which are available to the Keck community (Caltech, UC, NASA, UH, \& NOAO) through the standard proposal process*. These include $\mathrm{H}$ $(1.6 \mu \mathrm{m}), \mathrm{K}(2.2 \mu \mathrm{m})$, and L-band $(3.5 \mu \mathrm{m})$ visibility-amplitude modes at a variety of spectral resolutions (see Ragland et al, this volume for more details), and the $10 \mu \mathrm{m}$ nulling mode (see Colavita et al, this volume). With a grant from the NSF MRI program, Keck Observatory is continuing development of its ASTRA (ASTrometric and phase-Referenced Astronomy) project to add additional observational capabilities. ASTRAs self-phasereferenced high-spectral-resolution mode is currently available to the Keck community, the dual-field mode is entering the shared-risk-observing phase and the astrometric model is under development (Woillez et al, this volume). For a description of the science enabled by this mode, see the papers by Eisner and Pott in this volume.

\section{SCIENCE OVERVIEW}

Over the last two years, the science pursued by observers using KI has ranged from nulling observations of the closest main sequence stars and the formation of stars and planets to the energetic world of novae and the centers of active galactic nuclei. The peer-reviewed science papers using KI data since 2008 are:

- Luminosity-variation Independent Location of the Circum-nuclear, Hot Dust in NGC 4151 Authors: Pott, Jorg-Uwe, et al, 2010, The Astrophysical Journal, 715, 736.

- Ruling out Stellar Companions and Resolving the Innermost Regions of Transitional Disks with the Keck Interferometer, J.-U. Pott et al, 2009, The Astrophysical Journal, 710, 265.

\footnotetext{
Further author information: Send correspondence to R.A.: E-mail: rla@ipac.caltech.edu

*see http://nexsci.caltech.edu/software/KISupport/ for more details
}

Optical and Infrared Interferometry II, edited by William C. Danchi, Françoise Delplancke, Jayadev K. Rajagopal, Proc. of SPIE Vol. $7734,77340 C \cdot$ C 2010 SPIE · CCC code: 0277-786X/10/\$18 - doi: 10.1117/12.856494 
- Exploring the inner region of Type 1 AGNs with the Keck interferometer, M. Kishimoto et al, 2009, Astronomy \& Astrophysics, 507, 3, L57-L60.

- Interferometric Evidence for Resolved Warm Dust in the DQ Tau System, A. Boden et al, 2009, Astrophysical Journal Letters, 696, 2, L111-L114.

- Spatially Resolved Spectroscopy of Sub-AU-Sized Regions of T Tauri and Herbig Ae/Be Disks, J.A. Eisner et al, 2009, Astrophysical Journal, 692, 1.

- 51 Ophiuchus: A Possible Beta Pictoris Analog Measured with the Keck Interferometer Nuller, C.C. Stark et al, 2009, Astrophysical Journal, 703, 2, 1188-1197.

- First L-Band Interferometric Observations of a Young Stellar Object: Probing the Circumstellar Environment of MWC 419, S. Ragland et al, 2009, Astrophysical Journal, 703, 1, 22-29.

- Keck Interferometer Nuller Data Reduction and On-Sky Performance, M.M. Colavita et al, 2009, PASP, $121,884,1120-1138$.

- Milliarcsecond N-Band Observations of the Nova RS Ophiuchi: First Science with the Keck Interferometer Nuller, R. Barry et al., 2008, Astrophysical Journal, 677, 1253.

- Preliminary Orbit of the Young Binary Haro 1-14c, G. Schaefer et al, 2008, The Astronomical Journal, Volume 135, Issue 5, pp. 1659-1668.

- A Tale of Two Herbig Ae Stars, MWC 275 and AB Aurigae: Comprehensive Models for Spectral Energy Distribution and Interferometry, A. Tannirkulam, et al, 2008, Astrophysical Journal, 689, 1, 513-531.

- Strong Near-Infrared Emission Interior to the Dust Sublimation Radius of Young Stellar Objects MWC 275 and AB Aurigae, A. Tannirkulam, et al, 2008, Astrophysical Journal, 677, 1, L51-L54.

In the sections below, we describe highlights from some of these works.

\section{MATERIAL AROUND NEARBY MAIN SEQUENCE STARS}

One of the primary goals in NASA's development of KI was a nulling mode to survey nearby main sequence stars for exozodical dust. These stars are potential targets for future planet finding and characterization missions and a high level of exozodical dust can hide the signature of Earth-like planets. The detection of low level emission around a bright point source is a problem of dynamic range rather than pure sensitivity and the KI nuller was specifically designed to address this challenge. The nuller hardware is described in Colavita et al $(2008)^{1}$ and the performance and data pipeline in Colavita et al (2009) $)^{2}$ and Colavita et al (this volume).

Observations of the nearby main sequence targets were conducted as the NASA Exo-zodical Dust Survey Key Project by three competitively selected teams led by P. Hinz (University of Arizona), M. Kuchner (Goddard Space Flight Center) and E. Serabyn (Jet Propulsion Laboratory). The observations were conducted from 2008 February to 2009 January over 32 nights and observed 44 unique targets (a list of the targets is available at http://nexsci.caltech.edu/software/KISupport/KeyScienceTargets.shtml). Analysis of the complete data set by the KI team found an overall performance level in the null uncertainty of $0.3 \%(1 \sigma)$ for sources brighter than 2 $\mathrm{Jy}^{2}{ }^{2}$ Here we briefly summarize some results from the Key Science teams.

The Hinz team combined the KI data with Spitzer and MMT nulling data to characterize any circumstellar material over a wide range of radii. For beta Leo, the KI data, along with the other observations, show a series of radial rings of material (Stock et al, 2010, submitted). For omicron Leo and alpha CrB, the detected KI excesses most likely arise from the binary companions rather than from circumstellar dust (Stock et al, 2010, submitted).

The Serabyn team is analyzing a sample primarily consisting of non-detections for low-dust stars (as an example see Figure 1), from which to derive zodi levels upper limits. The team has established the following method: (a) use the spectral data which provides the highest combined SNR $(8-9 \mu \mathrm{m})$, (b) compute 1-zodi brightness around each star using ZODIPIC, ${ }^{3}$ (c) for each individual observation (i.e. for the precise sky orientation of the 
KI Nuller fringes) compute the predicted leak, where 1-zodi is defined as having the same optical depth at $1 \mathrm{AU}$ as in the solar system zodi, (d) scale the number of zodis until it matches the measured leak excess (i.e. measured leak minus the contribution from the photosphere of the central star), and repeat for measured leak +- error, (e) average the results and combine the errors (formal and external) from multiple observations for each target as described in, ${ }^{2}$ and (f) repeat for a range of exo-zodi cloud orientations (inclination and PA). The limits derived will represent a significant improvement in our level of knowledge about the level of exo-zodi emission around nearby main sequence stars, with respect to that afforded by the spectro-photometric techniques more typically used. The statistics of these upper limits will provide meaningful extrapolation into the regime of interest to future terrestrial planet finding missions.
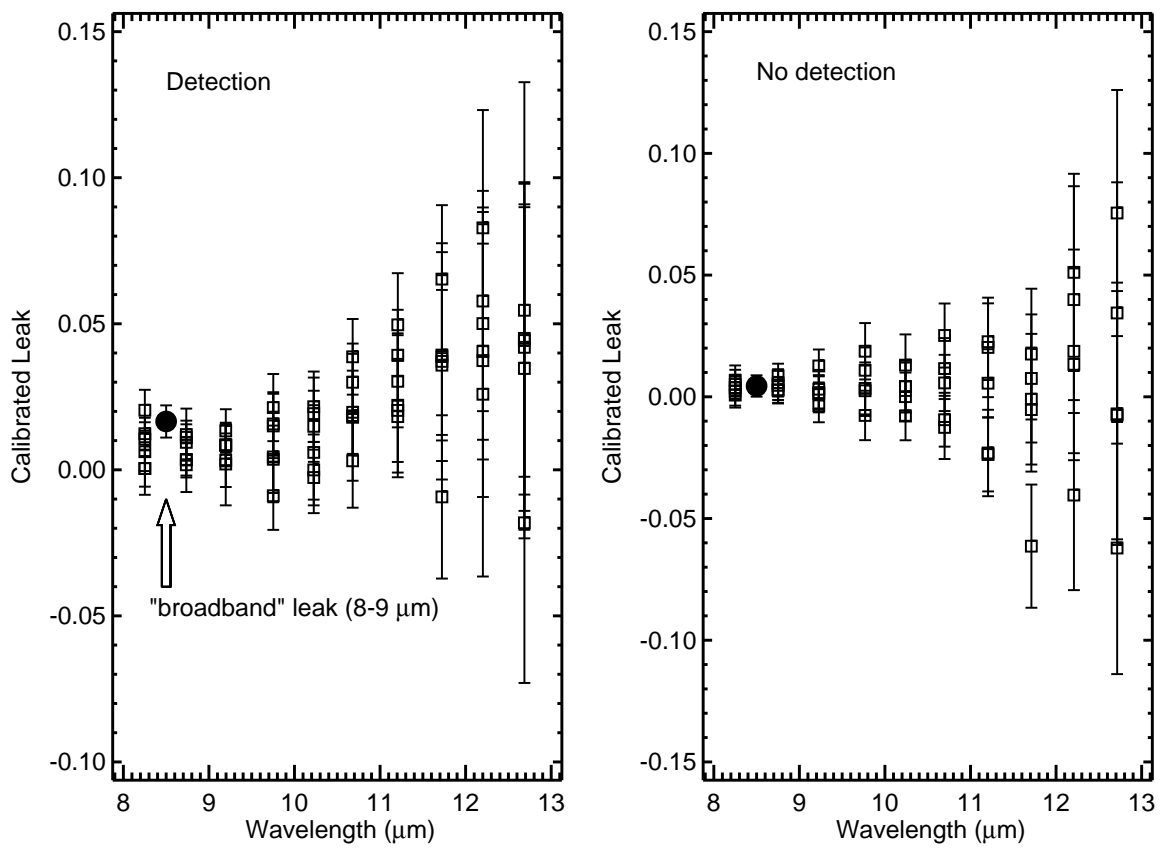

Figure 1. Example calibrated leak data for two objects, showing a detection and non-detection in the highest SNR broadband channel $(8-9 \mu \mathrm{m})$ (from Millan-Gabet et al, 2010, in preparation).

\subsection{Nulling calibrator stellar diameters}

One astrophysical product of the nulling Key Science campaign was measured K-band angular diameters for the relatively bright stars used as calibrators. Although the majority of the stars used as calibrators have spectral types earlier than K5 (99 of 120), many (21 of 120) are giants of later spectral type, for which angular diameter estimates based on model stellar atmospheres are especially unreliable. Since the nuller has its optical path stabilized via K-band fringe trackers, this data can be used to directly determine the stellar visibility and then the angular diameters. ${ }^{2}$ The key science targets, which were all well-characterized main sequence stars were used as calibrators for this analysis. A few of the key science targets are known to have circumstellar dust which contributes $1-2 \%$ of the K-band flux; however, including this component changes the diameters derived for the calibrators by at most 5\%. For the calibrators used for key science targets that are known to be binaries, this procedure could not be used. In those cases (13 of 120), the calibrators were used to calibrate each other, assigning to each of them in turn the role of target, and adopting for the others angular diameters from the surface-brightness relations of Bonneau et al. $(2006)^{4}$ with conservative $10 \%$ errors.

For each calibrator, a uniform disk diameter was fit to all the K-band visibility amplitude data available. These uniform disk diameters were converted to limb-darkened diameters using the standard formula for linear limb darkening. ${ }^{5}$ The resulting angular diameters at both $\mathrm{K}$ and N-band are given in Ref. ${ }^{2}$ Figure 2 shows the 
resulting limb-darkened diameters against the diameters predicted by the surface-brightness relations of Ref. ${ }^{4}$ Both estimates correlate well, validating the conversion from measured K-band to N-band diameters.

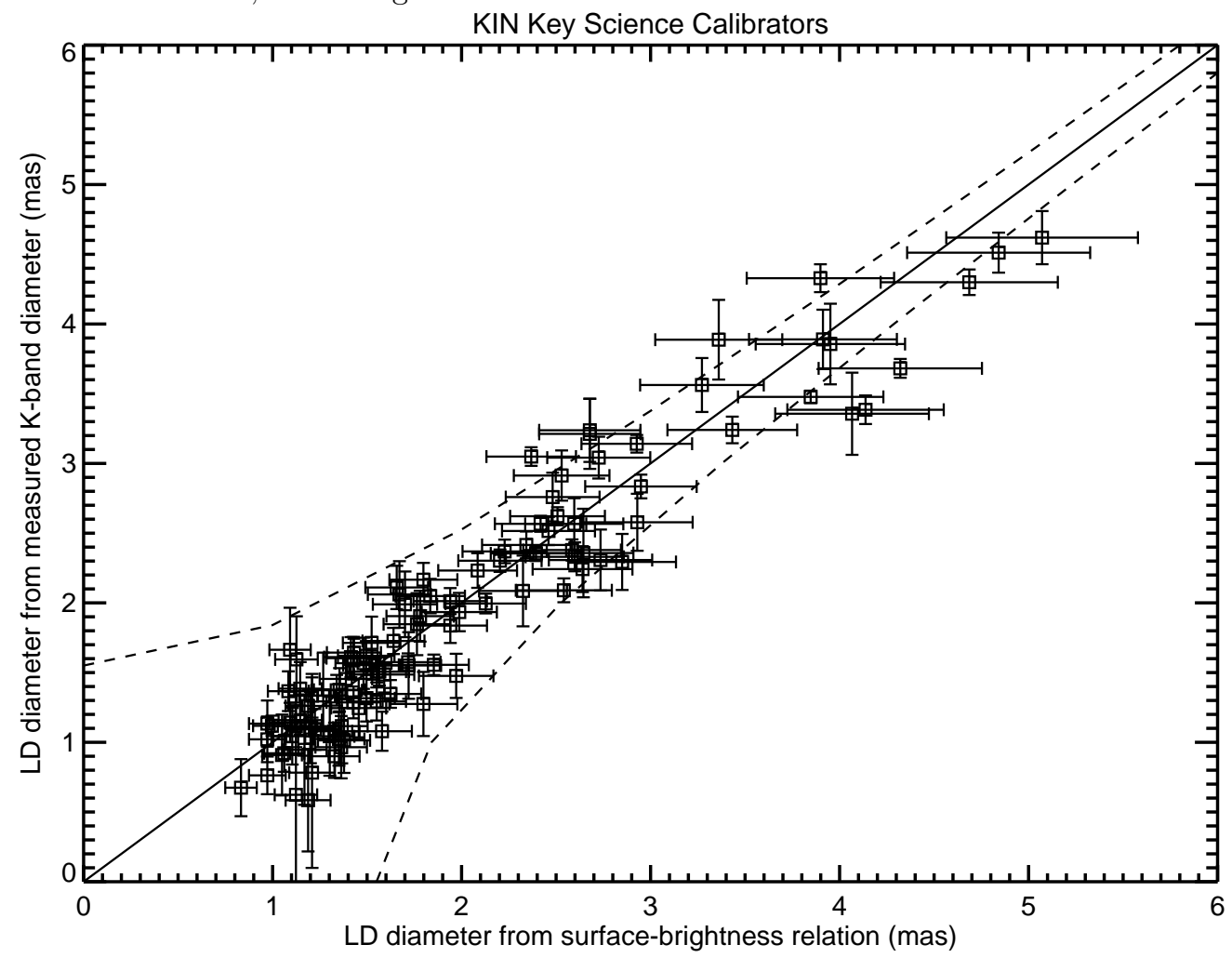

Figure 2. Limb-darkened diameters from K-band measurements vs. predictions from the surface-brightness relations of Bonneau et al. (2006) for the calibrator stars of the key science program. The dashed lines correspond to diameter errors which would cause a $0.2 \%$ change in leakage at $10 \mu \mathrm{m}$. Figure from Colavita et al (2009).

\section{YOUNG STELLAR OBJECTS}

Many of the studies with KI focus on the structure of circumstellar material around young stellar objects as the angular scales probed by infrared interferometry are well suited for the inner disk. The first observations using the L-band mode were of the massive young stellar object MWC 419. ${ }^{6}$ These observations also included medium resolution ( $\mathrm{R} \sim 230) \mathrm{K}$-band data taken simultaneously. Using geometric models, Ragland et al (2009) found that the apparent size increases linearly with wavelength from the $\mathrm{K}$ to $\mathrm{L}$ bands (2 to $4 \mu \mathrm{m}$ ) (Figure 3), suggesting that the disk is extended with a temperature gradient. A model with a radial power-law temperature gradient fit to both the interferometric measurements and the spectral energy distribution has a power-law slope close to that expected from an optically thick disk.

A second area in young stellar objects in which KI has substantially contributed is determination of binary orbits. There are relatively few direct mass measurements for young stars less than 1 solar mass and accurate measurements can be used to calibrate the theoretical evolutionary tracks. Observations of the spectroscopic binary DQ Tau were combined with radial velocity measurements from the literature to constrain the visual orbit $^{7}$ (Figure 4). The KI data of Boden et al (2009) exhibit a near-infrared excess which arises from a size scale close to that of the binary (0.2 AU) rather than the inner edge of the circumbinary disk (0.4 AU). This supports the hypothesis proposed by previous authors that warm material exists near the binary.

The sensitivity of KI has allowed studies of young stellar objects to push to fainter objects previously not studied with this technique. Pott et al $(2010)^{8}$ made observations of five transition disks, which are young objects thought to have optically thick outer disks, but optically thin inner disks. Their data exclude binaries with ux 


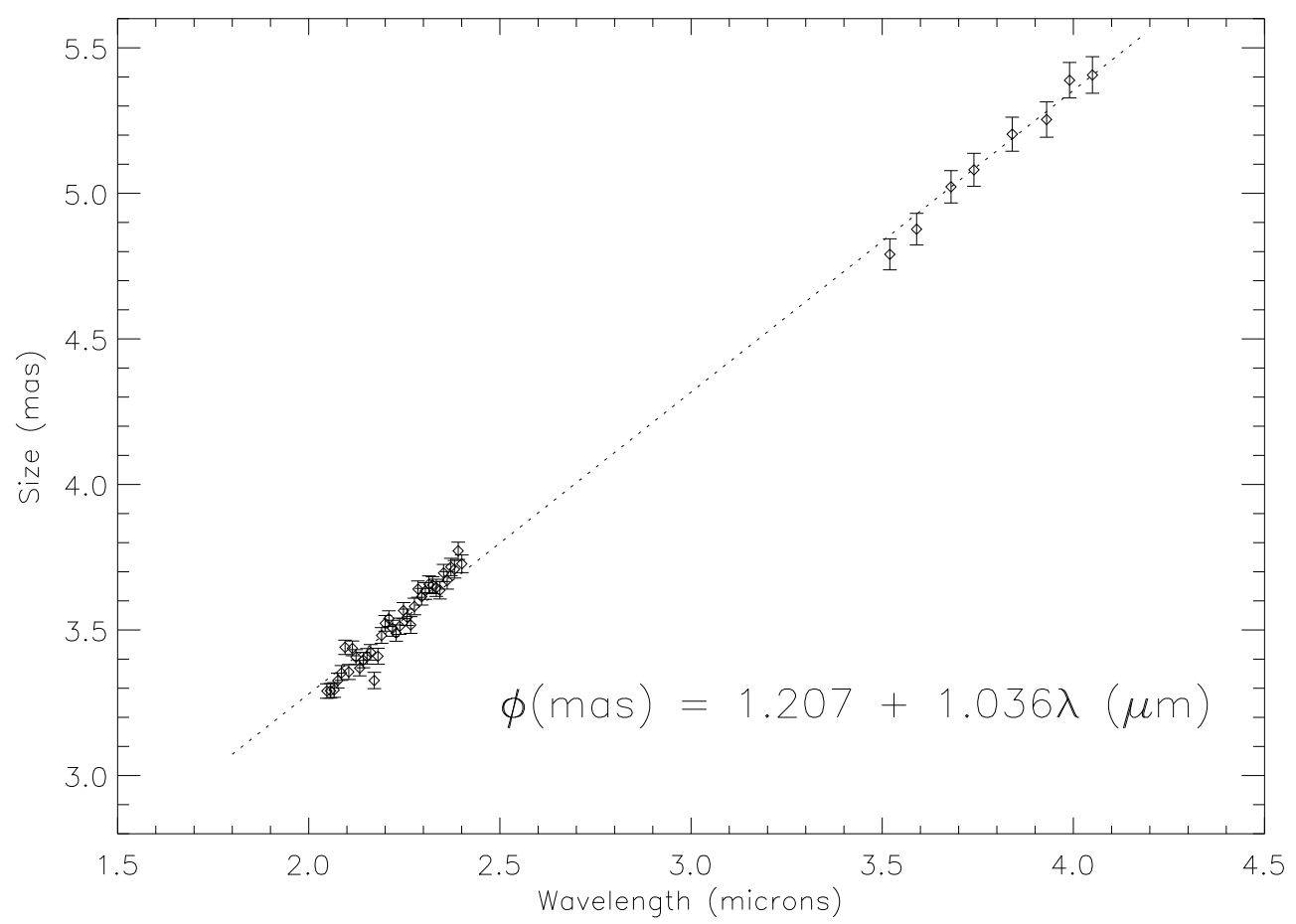

Figure 3. The derived uniform-disk angular sizes as a function of wavelength are shown in diamond symbol along with error bars. A linear fit to these sizes (the dotted line) is also shown. Figure from Ragland et al (2009).

ratios of at least 0.05 and separations ranging from 2.5 to 30 mas $(0.354 \mathrm{AU})$ over $>94 \%$ of the area covered by the measurements as the cause for the cleared inner region. All but one of the targets were spatially resolved and the data were consistent with hot material on scales of $0.1 \mathrm{AU}$ which is separated from the cooler outer disk, consistent with SED modeling. See Figure 5 for an example of the ring model and excluded companion phase space for the object LkCa 15.

The new self-phase referenced mode (Woillez, this volume) enabled by the ASTRA instrument provides significantly higher spectral resolution in the K-band. Figure 6 shows a small section of the K-band near the Brackett gamma Hydrogen transition for the young stellar object MWC 1080 (Eisner et al 2010, submitted). These observations show that the Brackett gamma is more compact than the continuum and is consistent with a disk origin for the emission line.

\section{NOVAE}

Barry et al $(2008)^{9}$ observed the nova RS Oph with the nuller 3.8 days after the outburst which occured on 2006 February 12 and derived a size scale of 4.0 mas (FWHM) for a Gaussian profile. The data show evidence of enhanced neutral atomic hydrogen emission and atomic metals including silicon located in the inner spatial regime near the white dwarf ( WD) while the outer spatial region ( $\sim 17 \mathrm{AU})$ shows evidence for nebular emission lines and hot silicate dust. They suggest that these features have been excited by the nova in the outer spatial regime before the blast wave reached these regions, supporting a model in which the dust appears to be present between outbursts and is not created during the outburst event. They also discuss the results in terms of a unifying model of the system that includes an increase in density in the plane of the orbit of the two stars created by a spiral shock wave caused by the motion of the stars through the cool wind of the red giant star. 


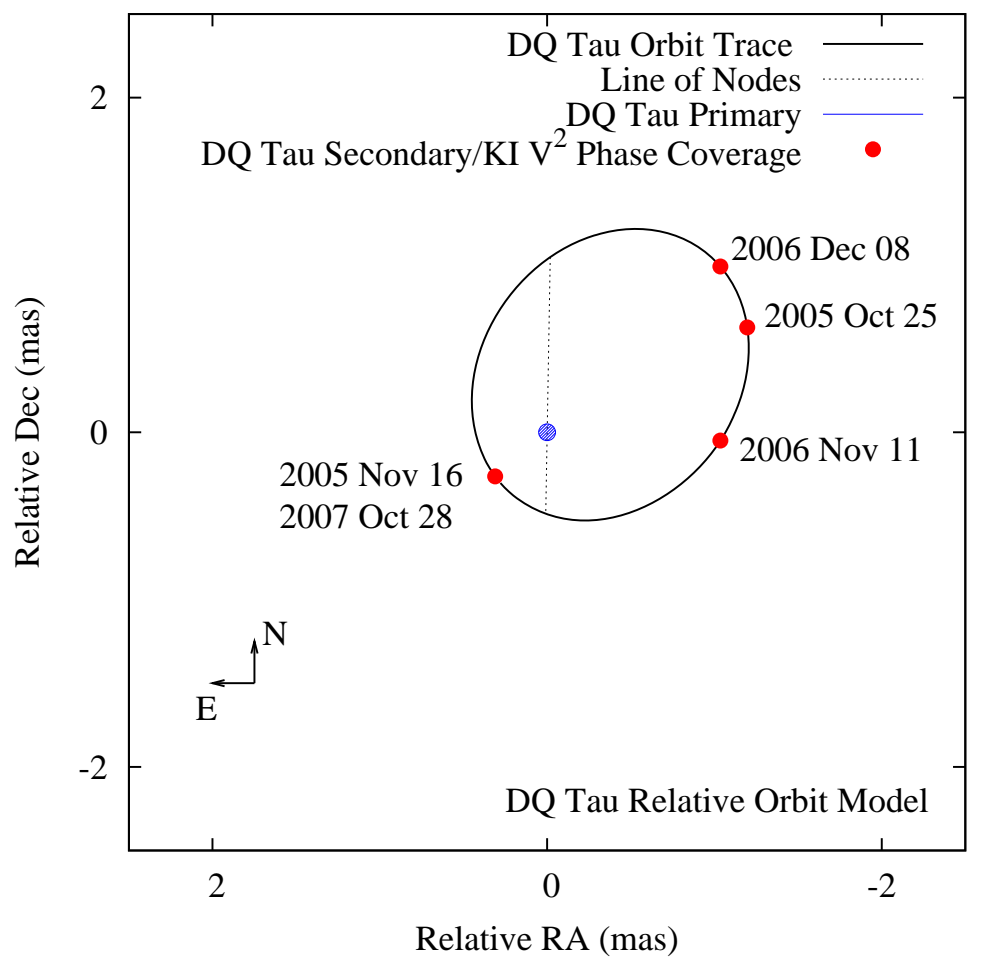

Figure 4. The relative visual orbit of the DQ Tau system, with the primary rendered at the origin, and the secondary position shown at the five epochs/orbit phases measured at KI. Figure from Boden et al (2009).

\section{ACTIVE GALACTIC NUCLEI}

The advent of large aperture interferometers (KI and VLTI) allowed the first observations of active galactic nuclei (AGN) in 2003. ${ }^{10,11}$ Recent improvements in K-band sensitivity (currently K magnitude $<10.3$ in the most senstivity mode) hve expanded the sample which can be observed.

Kishimoto et al (2009) have observed three type-1 AGN's and a QSO (quasi stellar object). ${ }^{12}$ All objects had a high measured visibility $\left(\mathrm{V}^{2} \sim 0.8-0.9\right)$. Using a ring model to measure the effective radius of the K-band emission, they find sizes roughly equal to or slightly larger than the reverberation radius as a function of AGN luminosity, suggesting that the KI observations are partially resolving the dust sublimation region.

A second team analyzed the multiple epochs of observations available for NGC $4151^{13}$ and found the measured visibilities from the three different nights are not correlated with the variations of the nuclear luminosity. Pott et al (2010) propose two models for the emission: 1) the visibility is dominated by emission from size scales smaller than $30 \mathrm{Mpc}$, which falls short of any dust reverberation measurement in NGC 4151 and of theoretical models of circum-nuclear dust distributions or 2) the visibility is dominated by hot dust ( $>1300 \mathrm{~K})$ at linear scales of about $50 \mathrm{Mpc}$. They favor the second hypothesis and also show that the KI data do not support a model where the dust emission size scale follows the nuclear variability of NGC 4151 as $\mathrm{R}_{\text {dust }} \propto L^{0.5}$.

\section{CONCLUSIONS}

Observations with the Keck Interferometer are being used to address a wide range of astrophysical questions, from circumstellar material around the closest stars to the disks around active galactic nuclei. The current capabilities of KI are described in Ragland et al (this volume) and are updated for each observing semester at http://nexsci.caltech.edu/software/KISupport/. Proposals to use KI can be submitted through any of the Keck community allocations (Caltech, UC, UH, NASA, NOAO/TSIP). 

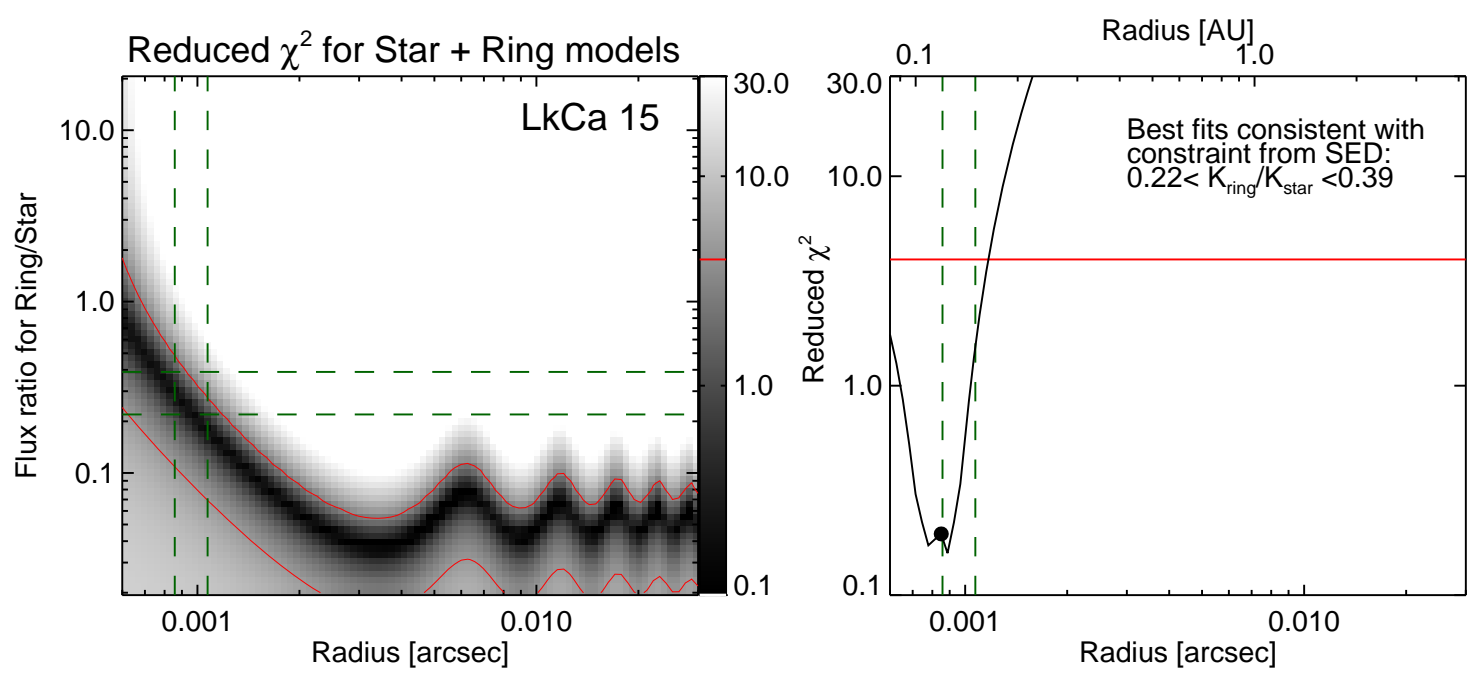

Figure 5. Star plus ring ts to the interferometric data of Lk Ca 15. The left panel shows the reduced $\chi^{2}$ for each set of model parameters. The lines in both panels trace the $99.7 \%$ condence contours; models outside these contours do not $\mathrm{t}$ the data, while models within these contours are possible based on the visibilities alone. The dashed lines indicate the ux ratio and inner radius range inferred from previous SED ts. Figure from Pott et al. (2010).
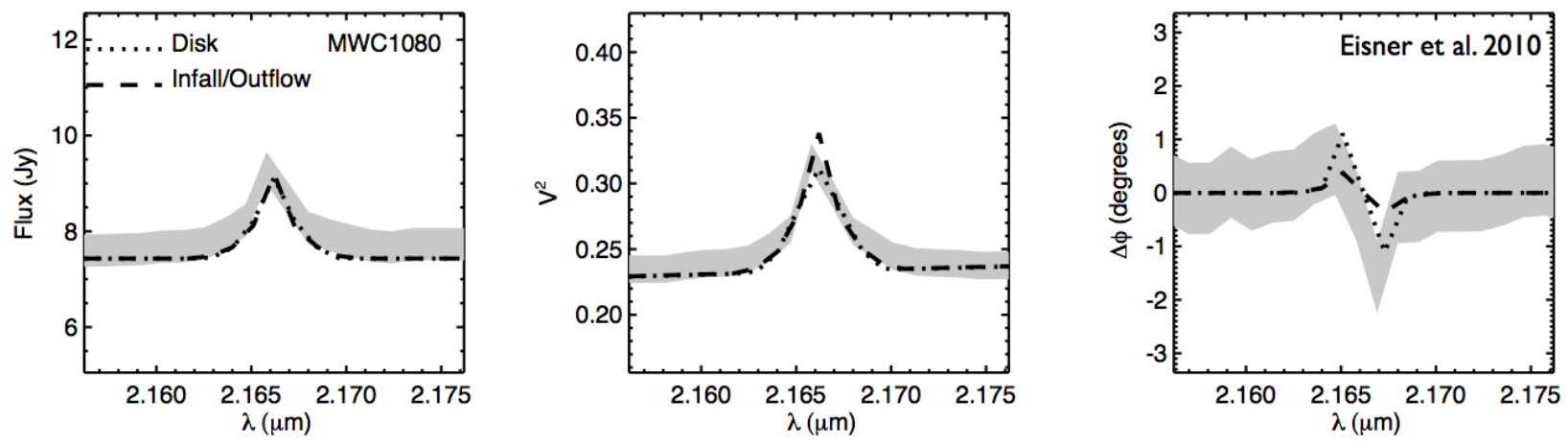

Figure 6. The flux, calibrated visibility and differential phase as a function of wavelength across the Brackett gamma line for the young stellar object MWC 1080. Figure from Eisner et al (2010, submitted).

\section{ACKNOWLEDGMENTS}

The Keck Interferometer is funded by NASA and developed and operated by the Jet Propulsion Laboraotry (California Institute of Technology), the W.M. Keck Observatory and the NASA Exoplanet Science Institute (California Institute of Technology). Observations presented were obtained at the W.M. Keck Observatory, which is operated as a scientific partnership amount the California Institute of Technology, the University of California and NASA. The Observatory was made possible by the generous financial support of the W.M. Keck Foundation. We would like to thank J.U.Pott, A. Boden, J. Eisner and N. Stock for providing material.

\section{REFERENCES}

[1] Colavita, M. M., Serabyn, E., Booth, A. J., Crawford, S. L., Garcia-Gathright, J. I., Ligon, E. R., Mennesson, B. L., Paine, C. G., Wizinowich, P. L., Ragland, S., Appleby, E. C., Berkey, B. C., Cooper, A., Dahl, W., Gathright, J. T., Hrynevych, M. A., Medeiros, D. W., Morrison, D., Panteleeva, T., Smith, B., Summers, K. R., Tsubota, K., Tyau, C., Wetherell, E., Woillez, J. M., Akeson, R. L., Millan-Gabet, R., Felizardo, 


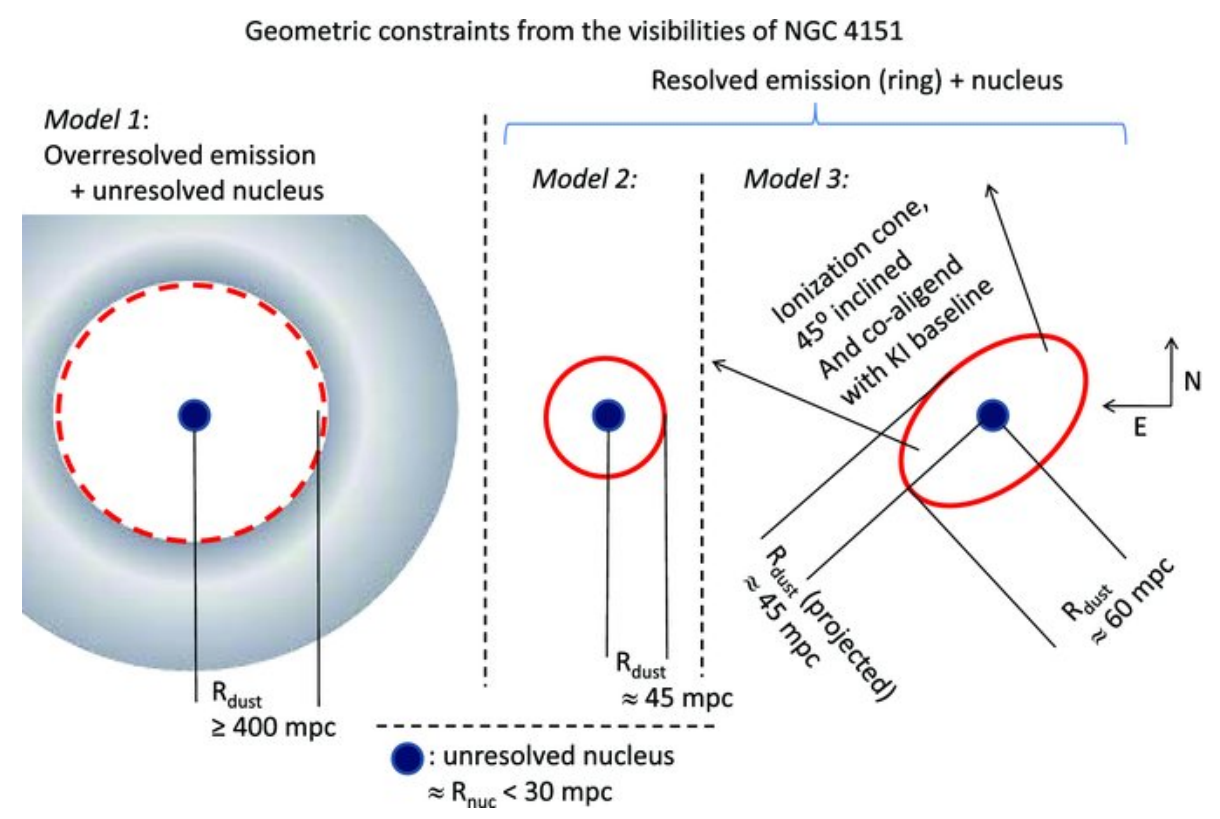

Figure 7. Sketches (not to scale) of the geometric constraints on the extended K-band emission of NGC 4151, seen through the 50 mas spatial filter of the Keck interferometer showing 3 different possible models. Figure from Pott et al (2010).

C., Koresko, C. D., and Herstein, J. S., "Keck Interferometer nuller update," in [Society of Photo-Optical Instrumentation Engineers (SPIE) Conference Series], Society of Photo-Optical Instrumentation Engineers (SPIE) Conference Series $\mathbf{7 0 1 3}$ (July 2008).

[2] Colavita, M. M., Serabyn, E., Millan-Gabet, R., Koresko, C. D., Akeson, R. L., Booth, A. J., Mennesson, B. P., Ragland, S. D., Appleby, E. C., Berkey, B. C., Cooper, A., Crawford, S. L., Creech-Eakman, M. J., Dahl, W., Felizardo, C., Garcia-Gathright, J. I., Gathright, J. T., Herstein, J. S., Hovland, E. E., Hrynevych, M. A., Ligon, E. R., Medeiros, D. W., Moore, J. D., Morrison, D., Paine, C. G., Palmer, D. L., Panteleeva, T., Smith, B., Swain, M. R., Smythe, R. F., Summers, K. R., Tsubota, K., Tyau, C., Vasisht, G., Wetherell, E., Wizinowich, P. L., and Woillez, J. M., "Keck Interferometer Nuller Data Reduction and On-Sky Performance," Proceedings of the Astronomical Society of the Pacific 121, 1120-1138 (Oct. 2009).

[3] Moran, S. M., Kuchner, M. J., and Holman, M. J., "The Dynamical Influence of a Planet at Semimajor Axis 3.4 AU on the Dust around $\epsilon$ Eridani," The Astrophysical Journal 612, 1163-1170 (Sept. 2004).

[4] Bonneau, D., Clausse, J., Delfosse, X., Mourard, D., Cetre, S., Chelli, A., Cruzalèbes, P., Duvert, G., and Zins, G., "SearchCal: a virtual observatory tool for searching calibrators in optical long baseline interferometry. I. The bright object case," Astronomy and Astrophysics 456, 789-789 (Sept. 2006).

[5] Hanbury Brown, R., Davis, J., Lake, R. J. W., and Thompson, R. J., "The effects of limb darkening on measurements of angular size with an intensity interferometer," Monthly Notices of the Royal Astronomical Society 167, 475-484 (June 1974).

[6] Ragland, S., Akeson, R. L., Armandroff, T., Colavita, M. M., Danchi, W. C., Hillenbrand, L. A., MillanGabet, R., Ridgway, S. T., Traub, W. A., Vasisht, G., and Wizinowich, P. L., "First L-Band Interferometric Observations of a Young Stellar Object: Probing the Circumstellar Environment of MWC 419," The Astrophysical Journal 703, 22-29 (Sept. 2009).

[7] Boden, A. F., Akeson, R. L., Sargent, A. I., Carpenter, J. M., Ciardi, D. R., Bary, J. S., and Skrutskie, M. F., "Interferometric Evidence for Resolved Warm Dust in the DQ Tau System," The Astrophysical Journal Letters 696, L111-L114 (May 2009).

[8] Pott, J., Perrin, M. D., Furlan, E., Ghez, A. M., Herbst, T. M., and Metchev, S., "Ruling Out Stellar Companions and Resolving the Innermost Regions of Transitional Disks with the Keck Interferometer," The Astrophysical Journal 710, 265-278 (Feb. 2010). 
[9] Barry, R. K., Danchi, W. C., Traub, W. A., Sokoloski, J. L., Wisniewski, J. P., Serabyn, E., Kuchner, M. J., Akeson, R., Appleby, E., Bell, J., Booth, A., Brandenburg, H., Colavita, M., Crawford, S., CreechEakman, M., Dahl, W., Felizardo, C., Garcia, J., Gathright, J., Greenhouse, M. A., Herstein, J., Hovland, E., Hrynevych, M., Koresko, C., Ligon, R., Mennesson, B., Millan-Gabet, R., Morrison, D., Palmer, D., Panteleeva, T., Ragland, S., Shao, M., Smythe, R., Summers, K., Swain, M., Tsubota, K., Tyau, C., Wetherell, E., Wizinowich, P., Woillez, J., and Vasisht, G., "Milliarcsecond N-Band Observations of the Nova RS Ophiuchi: First Science with the Keck Interferometer Nuller," The Astrophysical Journal 677, 1253-1267 (Apr. 2008).

[10] Swain, M., Vasisht, G., Akeson, R., Monnier, J., Millan-Gabet, R., Serabyn, E., Creech-Eakman, M., van Belle, G., Beletic, J., Beichman, C., Boden, A., Booth, A., Colavita, M., Gathright, J., Hrynevych, M., Koresko, C., Le Mignant, D., Ligon, R., Mennesson, B., Neyman, C., Sargent, A., Shao, M., Thompson, R., Unwin, S., and Wizinowich, P., "Interferometer Observations of Subparsec-Scale Infrared Emission in the Nucleus of NGC 4151," The Astrophysical Journal Letters 596, L163-L166 (Oct. 2003).

[11] Jaffe, W., Meisenheimer, K., Röttgering, H. J. A., Leinert, C., Richichi, A., Chesneau, O., Fraix-Burnet, D., Glazenborg-Kluttig, A., Granato, G., Graser, U., Heijligers, B., Köhler, R., Malbet, F., Miley, G. K., Paresce, F., Pel, J., Perrin, G., Przygodda, F., Schoeller, M., Sol, H., Waters, L. B. F. M., Weigelt, G., Woillez, J., and de Zeeuw, P. T., "The central dusty torus in the active nucleus of NGC 1068," Nature 429, 47-49 (May 2004).

[12] Kishimoto, M., Hönig, S. F., Antonucci, R., Kotani, T., Barvainis, R., Tristram, K. R. W., and Weigelt, G., "Exploring the inner region of type 1 AGNs with the Keck interferometer," Astronomy and Astrophysics 507, L57-L60 (Dec. 2009).

[13] Pott, J., Malkan, M. A., Elitzur, M., Ghez, A. M., Herbst, T. M., Schödel, R., and Woillez, J., "Luminosityvariation Independent Location of the Circum-nuclear, Hot Dust in NGC 4151," The Astrophysical Journal 715, 736-742 (June 2010). 